\title{
Classification of methods of moving the scanning sensor of a mechatronic profiler along the trajectories of plane curves
}

\author{
Sergey Vasiliev ${ }^{1,2}$ \\ ${ }^{1}$ Chuvash State University named after I.N. Ulyanov, Cheboksary, 428015, Russia \\ ${ }^{2}$ Nizhny Novgorod State University of Engineering and Economics, Knyaginino, 606340, Russia
}

\begin{abstract}
The paper considers using the plane curves analysis to classify the methods of moving the scanning sensor of a mechatronic profiler. The main purpose of the study is to determine the possibility of classifying the methods of moving the scanning sensor of a mechatronic profiler along the trajectories of plane curves using the characteristics obtained by analyzing the plain curves. According to the Archimedes spiral curve a large number of point clouds will be concentrated in the central part of the area under study that makes the study time-consuming as a whole. It is necessary to specify an optimal trajectory of the profiler sensor movement along the calculated plane curve to study of the surface thoroughly. It was found that using the trajectory of the sensor movement along the Fermat spiral is more promising, since the number of points scanned in the center is less and will be proportional to the number of points on the elementary plots evenly distributed on the area under study. The classification of methods of moving the scanning sensor of a mechatronic profiler was made on the basis of the results obtained in studying the trajectories of plain curves.
\end{abstract}

\section{Keywords}

Mechatronic profiler, scanning sensor

\section{Introduction}

The paper considers using the plane curves analysis to classify the methods of moving the scanning sensor of a mechatronic profiler. The main purpose of the study is to determine the possibility of classifying the methods of moving the scanning sensor of a mechatronic profiler along the trajectories of plane curves using the characteristics obtained by analyzing the spiral movement of the sensor.

The field mechatronic profiler allows the laser sensor to move along the specified trajectories of plain curves, mainly in spirals $[1,2,3]$. Spiral (french-"spirale", latin - "spira" mean "spire") is a plain curve that usually goes around one or several points, moving to or away from them. The spirals can be algebraic and pseudospirals. Algebraic spirals are the ones whose equation in polar coordinates is algebraic relative to the variables $r$ and $\mathrm{j}$. Algebraic spirals include: hyperbolic spiral, Archimedean spiral, Galilean spiral, Fermat spiral, parabolic spiral, lituus. Pseudospirals are apirals. Their natural equations take into account the radius of curvature and the arc length. For $m=1$, the pseudospiral is a logarithmic spiral, for $m=-1$ is Cornu spiral, and for $m=1 / 2$, the evolvent of circle.

The existing mechatronic profiler $[3,4,5,6]$, consists of a massive base, a level, an angle sensor, an electronic unit, a laptop, a movable arm with a counterweight, a laser position sensor, a screw mechanism with a carriage, electric motors, and a cylindrical transmission. The existing profiler and the newly developed one have some similar components that include a base, a rack, an angle sensor, a housing, a power supply unit, a control unit, a support wheel, a satellite, a field laptop, a laser sensor, a screw, and electric motors. The disadvantage of the existing mechatronic profiler is its design that

III International Workshop on Modeling, Information Processing and Computing (MIP: Computing-2021), May 28, 2021, Krasnoyarsk, Russia

EMAIL: vsa_21@mail.ru (Sergey Vasiliev)

ORCID: 0000-0003-3346-7347 (Sergey Vasiliev)

(c) (i) (C) 2021 Copyright for this paper by its authors.

Use permitted under Creative Commons License Attribution 4.0 International (CC BY 4.0).

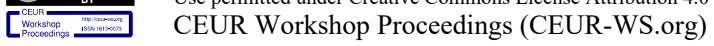


makes the process of surface profiling technologically difficult because of various cables connecting the electronic signal processing unit, sensors, electric motors, and a laptop when the profiler performs a large number of turns.

\section{Materials and methods}

A field mechatronic profiler was developed in the Laboratory of Precision Mechanics and Robotics of the Chuvash State University named after I. N. Ulyanov [7,8,9] (Figure 1:).

The field mechatronic profiler consists of a base with mounting rods, a rack on which an angle sensor is installed, a housing with a power supply unit and a control unit placed in it, a fixed support wheel 8 , a satellite communicating with it, a field laptop, a guide, a carriage, a laser position sensor, a screw, electric motors, GPS receiver, an accelerometer, a gyroscope, a compass, a thermohygrometer, and a soil moisture meter. The field laptop is provided with the information measurement system and computer control to coordinate the operation of electric motors during the measurement, as well as with a program for processing the data received from the sensors and devices. The housing is mounted on a rack by means of rolling bearings. Fixed support wheel, a satellite and a motor located on the movable housing are used to rotate it. The communication of the field laptop with the control unit, sensors, electric motors, GPS receiver and measuring devices is provided via a Bluetooth connection using Bluetooth radio modules built into the laser sensor, control unit and laptop.

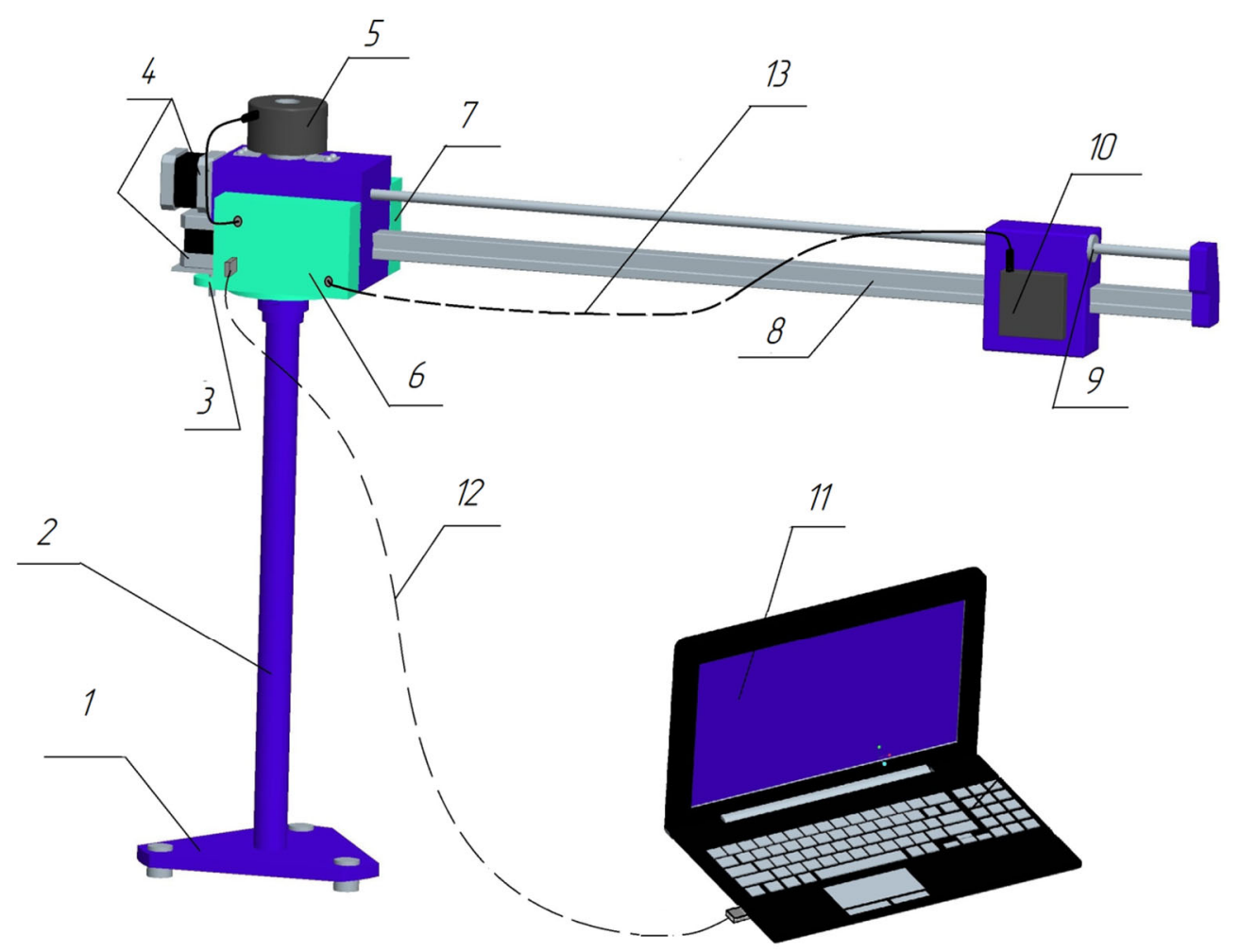

Figure 1: Field mechatronic profiler of parallel-serial structure: 1 -base, 2 - rack, 3 - housing, 4 electric motors, 5 - angle sensor (encoder), 6 - control unit, 7 - power supply unit, 8 - guide, 9 carriage, 10 - laser position sensor, 11 - laptop, 12 - wireless Bluetooth connection of the laptop and the control unit, 13 - wireless Bluetooth connection of the laser position sensor and the control unit

\section{Results and discussion}

Considering the movement of the laser sensor in the horizontal plane, you can see that its movements are better described in polar coordinates. The position of the guide relative to the origin of coordinates should be taken as the original one, and the deviation from the original one should be set by the angle 
of turning. The movement of the laser sensor along the guide will be determined by the length of the beam from the origin/zero point of cordinates.

For example, in full coordinates, the equation of a circle of a given radius centered at $(0 ; 0)$ will take the form

$$
\left\{\begin{array}{c}
\rho=R \\
0<\varphi_{i}<360^{\circ} \\
z_{i}=H_{i}=0
\end{array}\right.
$$

where $\rho$ - is the radius of movement of the sensor in the $i$-th position, $\mathrm{m} ; \varphi_{i}-\mathrm{i}$ is the angle of turning of the sensor in the $i$-th position, deg.

This is a simple case of setting the sensor to move around a circle, but this trajectory also allows you to get enough information about the scanned surface. When the sensor moves around a circle in the horizontal plane, its laser beam will represent a generatrix moving around a circle, so that its other positions will constantly be parallel to the initial projection. We get a rough circular cylinder that is projected onto the scanned or studied surface. Assume that this surface is tilted at a certain angle, so in this case the projection of the beam will be a curve in the form of an ellipse. Finally, to determine the measurement path that is formed at the intersection of the tilted platform and a cylindrical surface with a measurement radius $\mathrm{R}$, we write the system of equations (2.15) in the polar coordinate system in the form:

$$
\left\{\begin{array}{c}
\rho=R, \\
0<\varphi_{i}<360^{\circ}, \\
z_{i}=H_{i}=\left(H_{x \max }-H_{0}\right) \cos \varphi_{i}+\left(H_{y \max }-H_{0}\right) \sin \varphi_{i}+H_{0} .
\end{array}\right.
$$

To convert into the Cartesian coordinate system, use the equations

$$
\left\{\begin{array}{l}
x=R \cos \gamma \\
y=R \sin \gamma \\
z=z
\end{array}\right.
$$

where $x$ - is the longitudinal coordinate, $\mathrm{m}$; is the transverse coordinate, $\mathrm{m} ; z$ - is the vertical coordinate - the height of the surface irregularities at a given point, $\mathrm{m}$.

To determine the height of surface irregularities at a given point, use the expression

$$
z=z_{\max }-z^{\prime}
$$

where $z_{\max }$ - is the maximum distance between the position sensor and the surface, $\mathrm{m} ; z^{\prime}$ - is the actual distance between the position sensor and the surface at a given point, $\mathrm{m}$.

The scan in the Cartesian coordinate system can be obtained by a simple equation

$$
h=\operatorname{Rtg} \alpha \sin \gamma
$$

where $\alpha$-the slope of the surface under study, deg.; $\gamma$ - the angle of turning from the zero point of the laser sensor.

Consider the following guide curve which sets the trajectory of the sensor movement - the Archimedes spiral. The Archimedes spiral is determined by a plain curve and represents the trajectory of the laser sensor, which moves uniformly along the guide from the zero point, while the guide itself rotates uniformly around the axis of the device. The Archimedes spiral can be determined in full coordinates by the equation

$$
\left\{\begin{array}{l}
R=a+b \gamma \\
\gamma \in\left[\frac{a}{b}, \infty\right)
\end{array}\right.
$$

where $a$ - the coefficient, $b$ - the displacement of the laser sensor along the guide when it makes a turn equal to one radian.

If $a=0$, then

$$
\left\{\begin{array}{l}
R=b \gamma \\
\gamma \in[0, \infty)
\end{array}\right.
$$


The displacement of the laser sensor along the guide when it makes a turn equal to one radian is calculated by the expression

$$
b=\frac{s}{2 \pi}
$$

where $s-$ a spiral pitch.

According to formulas (7) and (8), we will convert the expression (6)

$$
h=\frac{s}{2 \pi} \gamma \operatorname{tg} \alpha \sin \gamma,
$$

Using the obtained formula (9), we draw the dependence of the distance change from the laser sensor to the tilted surface when scanning along the Archimedes spiral (Figure 2:). The sensor made 3.5 revolutions around the axis at a distance of $21 \mathrm{~cm}$ from it.

In this case, the length of the path made by the laser sensor can be determined by the expression

$$
L=\frac{s}{4 \pi}\left(\gamma \sqrt{1+\gamma^{2}}+\ln \left(\gamma+\sqrt{1+\gamma^{2}}\right)\right)
$$

Using the obtained expression, we draw a graphical dependence scan length increase along the Archimedes spiral (Figure 3:).

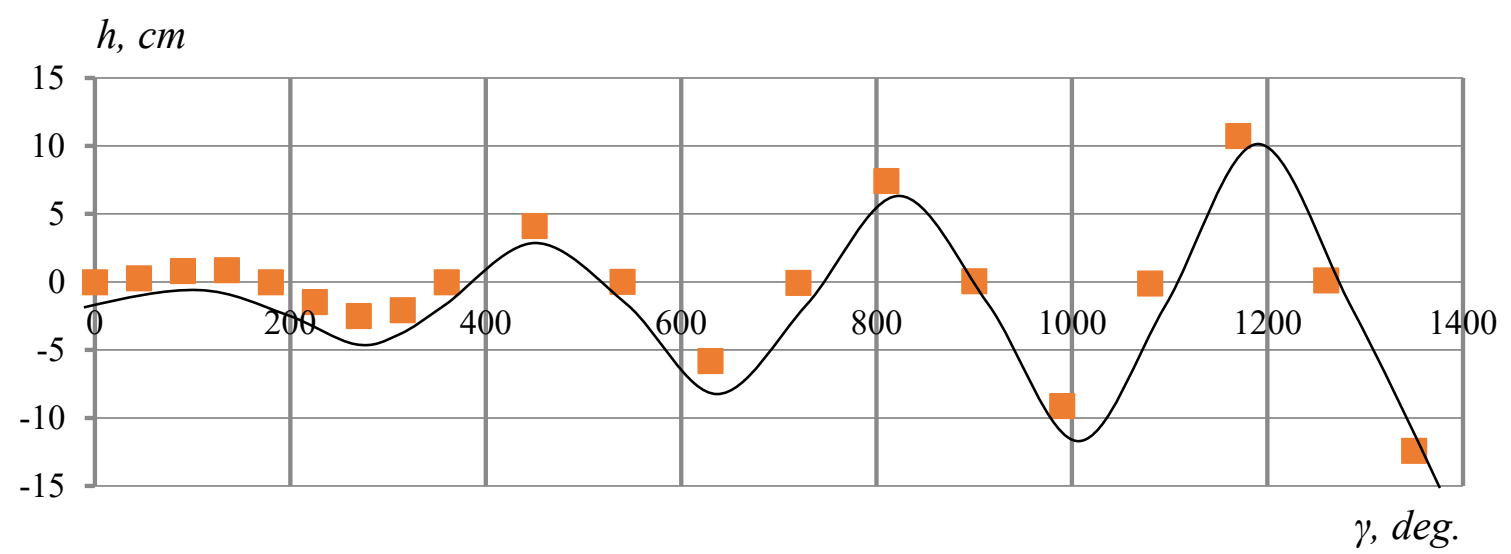

Figure 2: Dependence of the distance change from the laser sensor to the tilted surface when scanning along the Archimedes spiral

The analysis of the obtained graph allows us to conclude that according to the found dependence, a large number of point clouds will be concentrated in the central part of the area under study, which will make the study time-consuming.

Thus, to study of the surface thoroughly, it is necessary to find an optimal trajectory of the profiler sensor movement along the calculated plain curve. 


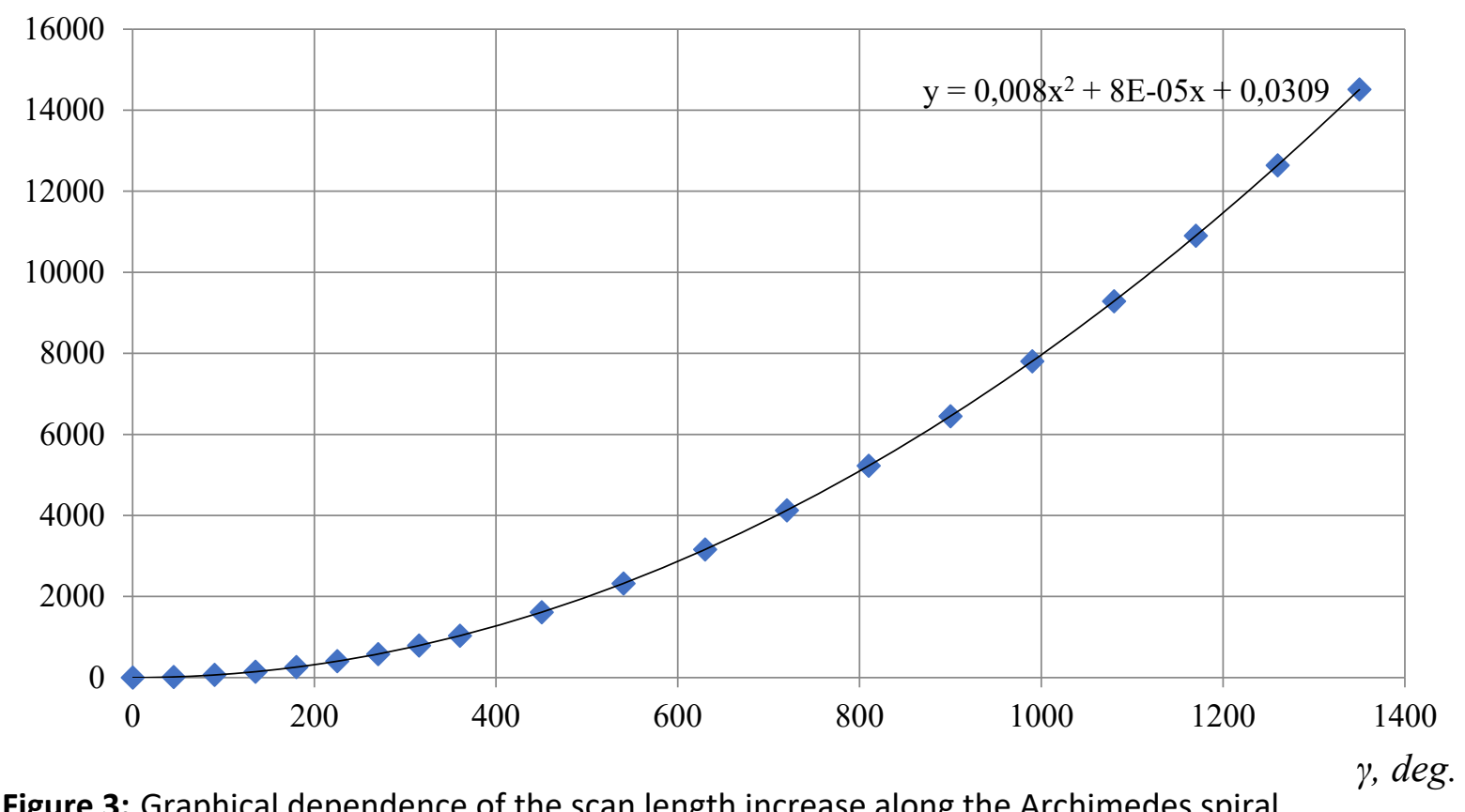

Figure 3: Graphical dependence of the scan length increase along the Archimedes spiral

Consider the following guide curve which sets the trajectory of the sensor movement - a Fermat spiral or a parabolic one. Let us present the well-known polar equation for the Fermat spiral (Figure 4:)

$$
R=\sqrt{s^{2} \gamma} .
$$

$h, \mathrm{~cm}$

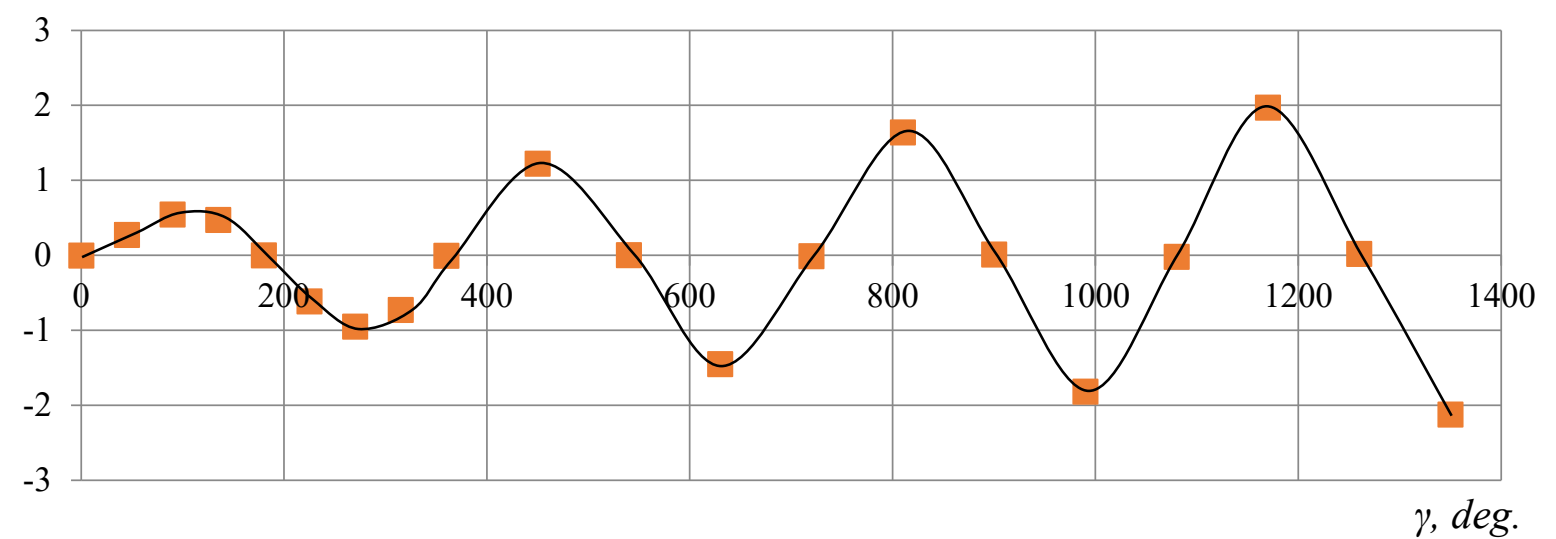

Figure 4: Dependence of the distance change from the laser sensor to the tilted surface when scanning along the Fermat spiral

The analysis of the data obtained allows us to conclude that using the trajectory of the sensor movement along the Fermat spiral it is more promising, since the number of points scanned in the center is less and will be proportional to the elementary plots evenly distributed on the area under study.

We will classify the methods of moving the scanning sensor of a mechatronic profiler (Figure 5:) based on the results obtained in studying the trajectories of plain curves. 


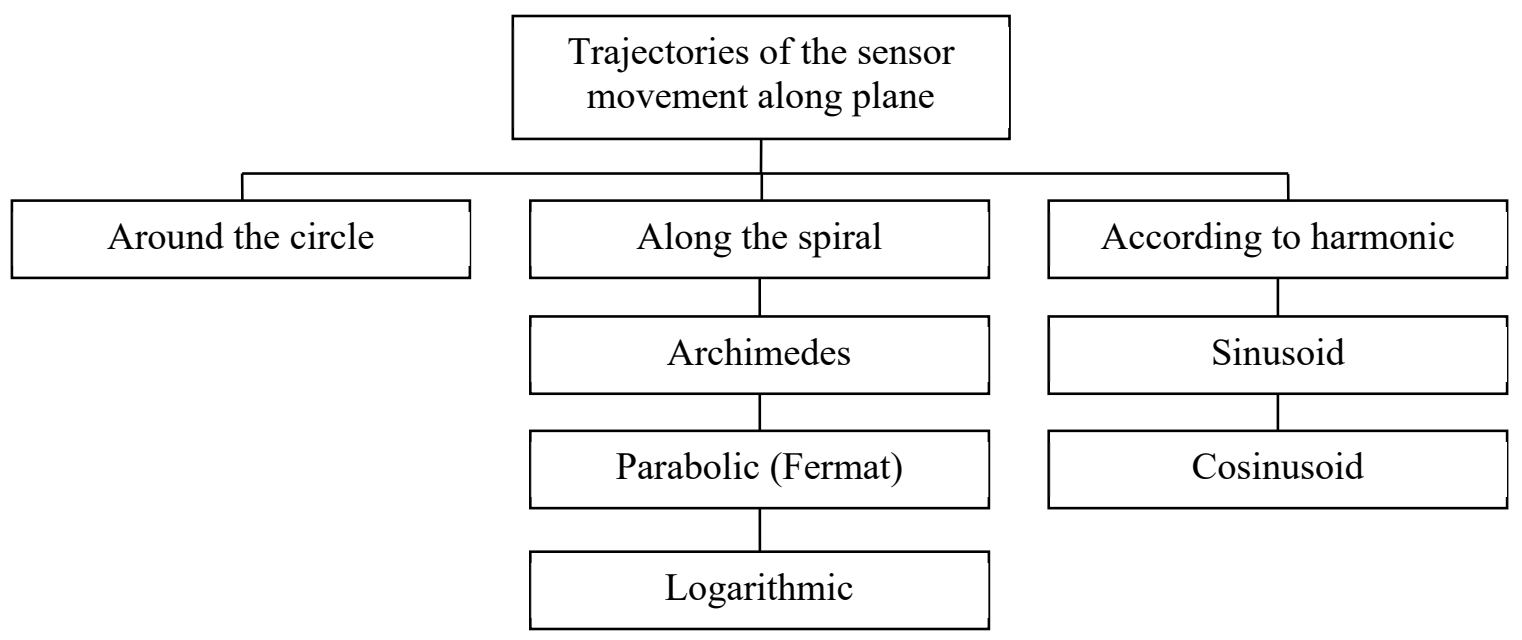

Figure 5: Classification of methods of moving the scanning sensor of a mechatronic profiler

The analysis of the developed classification of methods of moving the scanning sensor of a mechatronic profiler allows us to distinguish three main groups of plain curves. The profiler sensor provides the necessary information about the surface under study moving along these curves $[10,11$, 12].

\section{Conclusions}

The developed classification of methods of moving the scanning sensor can be used in the control programs of modern mechatronic profilers. The combination or sequential use of these trajectories will allow obtaining the necessary information in the quantitative and qualitative measurement of the surface under study with the possibility of creating a three-dimensional model.

\section{Acknowledgment}

The article was prepared under the financial support of the Russian Federation President grant no. MD-1198.2020.8.

\section{References}

[1] V. Alekseev, CEUR Workshop Proc 2258 (2018) 404-409.

[2] V. Alekseev, I. Ivanov, 25th Int. Conf. on Vacuum Technique and Technology 387012001 (2018).

[3] S. Vasiliev, A. Kirillov, I. Afanasieva, Engineering for Rural Development 12622616 (2018).

[4] S. Vasilyev, A. Vasilyev, M. Ivanov, A. Vasilyeva, IOP Conference Series: Materials Science and Engineering 450 (2019) 62018.

[5] I. Maksimov, A. Vasilyev, IOP Conference Series: Materials Science and Engineering 516012027 (2019).

[6] I. Maksimov, V. Maksimov, V. Alekseev, Simulation of Channel Development on the Surface of Agrolandscapes on Slopes, Eurasian Soil Science 49 (2016) 475-480.

[7] E. Alekseev, I. Maksimov, Investigation of seed uniformity under field and laboratory conditions, IOP Conf. Series: Materials Science and Engineering 450062008 (2018).

[8] S. Vasilyev, I. Maximov, A. Vasilyev, E. Vasilyeva, Elaborating of the device for the importation of liquid ameliorants into the soil, IOP Publishing IOP Conf. Series: Materials Science and Engineering 450062011 (2018).

[9] S. Vasiliev, R. Alexandrov, A. Fedorova, M. Vasiliev, S. Mishin, S. Limonov, Mechatronic profiler Pat. of the Russian Federation publ.23.06.2020 No 2724386 Bull. No 18 (2020). 
[10] I. Maksimov, V. Alekseev, S. Chuchkalov, Erosion resistance potential as a soil erosibility characteristic based on energy approach, IOP Conf. Series: Earth and Environmental Science 226 012067 (2019). DOI:10.1088/1755-1315/226/1/012067.

[11] V. Alekseev et al., Development of a criteria-based approach to agroecological assessment of slope agrolandscapes, Eastern-European Journal of Enterprise Technologies 6 10(96) (2018) 28-34. DOI:10. 15587/1729-4061.2018.148623.

[12] S. Chuchkalov, I. Fadeev, V. Alekseev, B. Mikhailov, Effect of Synthetic Detergents on Soil Erosion Resistance, KnE Life Sciences 5(1) (2020) 489-496. DOI:10.18502/kls.v5i1.6113. 